\title{
Decomposition Algorithms of Blocking the Selected Edges in the Digraph
}

\author{
Tsitsiashvili Gurami* \\ Institute for Applied Mathematics FEB RAS, Russia
}

*Corresponding author: Tsitsiashvili Gurami, Institute for Applied Mathematics FEB RAS, Vladivostok, Russia.

Received Date: October 17, 2019

Published Date: October 22, 2019

\begin{abstract}
In this paper, a protein network represented by a directed graph is considered. The problem of determining the minimum number of edges that break paths from the input proteins of the network to the output ones and passing through some subset of proteins in this network is analyzing. A decomposition algorithms are basing on a selection of classes of cyclically equivalent nodes in the sub-graphs with dedicated subset of nodes is suggesting.
\end{abstract}

Keywords: Cluster; Digraph; Sub-graph; Protein network; Connectivity component

\section{Concept}

By analogy with [1], we consider a protein network represented by a directed graph (digraph) $G$ with the set $U$ of nodes, which are proteins and whose directed edges are paired bonds between nodes represented in the Cytoscape program. Dedicate the subset $U^{\prime} \subseteq U$ of nodes and decrease in a comparison with [1] a number of edges, which block all paths from outside of the set $U^{\prime}$ outside of the set $U^{\prime}$.

Take all nodes from the subset $U^{\prime}$ and all edges between them. These nodes and edges create directed sub-graph $G^{\prime} \subseteq G$. Replace all (directed) edges of the sub-graph $G^{\prime}$ by undirected ones and obtain undirected graph $G^{\prime \prime}$. Define in the sub-graph $G^{\prime \prime}$ all its connectivity components $G^{\prime \prime}{ }_{1}, \ldots, G^{\prime \prime}{ }_{n}$. Return directions to all edges of the subgraph $G^{\prime \prime}{ }_{k}$ and define in such a way the sub-graph $G_{k}$ of the digraph $G, k=1, \ldots, n$.

Factorize each sub-graph $G_{k}$ by a relation of cyclic equivalence (two nodes are cyclically equivalent if there is a cycle containing both of them) and construct acyclic digraph with nodes - clusters of cyclic equivalence. Construct the partial order $\geq$ between the clusters (a relation $A \geq B$ is true if there is a way from cluster $A$ to cluster $B$ ) in $G_{k}$.

Denote by $V_{k}^{*}$ a set of edges incoming to $G_{k}$ and by $V_{k}^{* *}$ a set of edges out-coming from $G_{k}$ and designate by $p_{k}, q_{k}$ numbers of edges in these sets. Define the following sets $W_{k}^{*}, W_{k}^{* *}$ of clusters $G_{\mathrm{k}, \mathrm{i}} \in \mathrm{W}_{k}, \mathrm{i}=1, \ldots, \mathrm{m}_{k}$, in digraph $G_{k}$. Each cluster $G_{\mathrm{k}, \mathrm{i}} \in W_{k}^{* *}$ has final nodes of some edges from the set $V_{k}^{*}$. Similarly, each cluster $G_{\mathrm{k}, \mathrm{i}} \in W_{k}^{* *}$ has initial nodes of some edges from the set $V_{k}^{* *}$. The sets $W_{k}^{*}, W_{k}^{* *}$ may intersect.

Construct the set $\bar{W}_{k}{ }^{*}$, consisting of clusters $G_{\mathrm{k}, \mathrm{i}}$, such that $G_{\mathrm{k}, \mathrm{i}} \in W_{k}^{* * *}$ or $G_{\mathrm{k}, \mathrm{i}} \notin W_{k}^{* *}$, but there are some cluster $G_{\mathrm{k}, \mathrm{j}} \in W_{k}^{* *}$ and a way from $G_{\mathrm{k}, \mathrm{i}}$ to $G_{\mathrm{k}, \mathrm{j}}$ in $\operatorname{digraph} G_{k}$. By analogy construct the set $\bar{W}_{k}^{* *}$ of clusters $G_{\mathrm{k}, \mathrm{i}}$, such that $G_{\mathrm{k}, \mathrm{i}} \in W_{k}^{*}$ or $G_{\mathrm{k}, \mathrm{i}} \notin W_{k}^{*}$, but there are some cluster $G_{\mathrm{k}, \mathrm{j}} \in W_{k}^{*}$ and a way from $G_{\mathrm{k}, \mathrm{j}}$ to $G_{\mathrm{k}, \mathrm{i}}$ in $\operatorname{digraph} G_{k}$.

Designate by $\bar{V}_{k}^{*}$ all edges incoming some clusters containing in the set $\bar{W}_{k}^{*}$ from the outside of the graph $G_{k}$ (by $\bar{V}_{k}^{* *}$ all edges out-coming from some clusters in the set $W_{k}^{* *}$ outside the graph $G_{k}$ ). Denote $\overline{p_{k}} \leq p_{k}$ a number of edges in the set $\bar{V}_{k}{ }^{*}$ (denote $\overline{q_{k}} \leq q_{k}$ a number of edges in the set $\bar{V}_{k}^{* *}$ ).

Assume that $\bar{p}_{k} \leq \bar{q}_{k}$ (assume that $\bar{q}_{k} \leq \bar{p}_{k}$ ) and block all edges from $\bar{V}_{k}^{* *}$ (all edges from $\bar{V}_{k}^{*}$ ). In such a way, we block all paths from the outside of $G_{k}$ outside $G_{k}$ . Then a number of edges blocking the sub-graph $G_{k}$ equals' $\bar{r}_{k}=\min \left(\bar{p}_{k}, \bar{q}_{k}\right) \leq \min \left(\mathrm{p}_{k}, \mathrm{q}_{k}\right)=\mathrm{r}_{k}, \mathrm{k}=1, \ldots, \mathrm{n}$. Lastinequalities show, how this algorithm decreases a number of blocked edges in a 
comparison with [1]. Next step of the decomposition procedure in this algorithm may be an allocation of disconnected subsets in pairs of sets $W_{k}^{*}, W_{k}^{* *}, k=1, \ldots, n$.

\section{Acknowledgement}

Supported by Russian Fund of Basic Research's, project 17-0700177.

\section{Conflict of Interest}

No conflict of interest.

\section{References}

1. G Sh Tsitsiashvili (2019) Improved algorithm of blocking the selected nodes in the digraph, Annals of Biostatistics and Biometric Applications $3(3)$. 